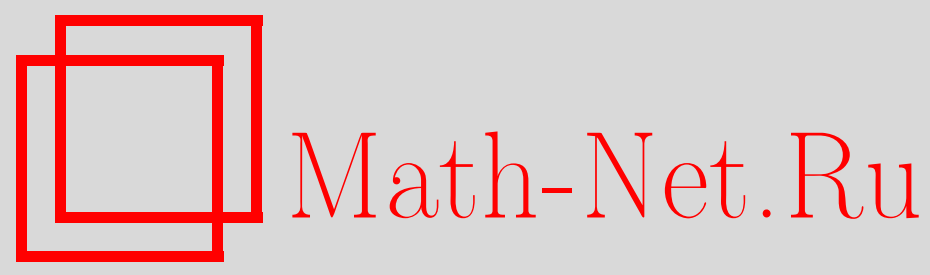

Ф. Н. Гарифьянов, Об одном разностном уравнении и его приложении к проблеме моментов, Матем. заметки, 2003, том 73, выпуск 6, 821-826

DOI: https://doi.org/10.4213/mzm229

Использование Общероссийского математического портала Math-Net.Ru подразумевает, что вы прочитали и согласны с пользовательским соглашением http://www.mathnet.ru/rus/agreement

Параметры загрузки:

IP : 54.224 .187 .69

26 апреля 2023 г., $17: 13: 41$

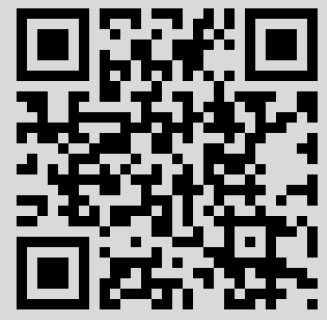




\section{ОБ ОДНОМ РАЗНОСТНОМ УРАВНЕНИИ \\ И ЕГО ПРИЛОЖЕНИИ К ПРОБЛЕМЕ МОМЕНТОВ}

\section{Ф. Н. Гарифьянов}

Исследовано разностное уравнение

$$
\sum_{m_{1}, m_{2}} \Omega\left[\sigma_{m_{1}, m_{2}}(z)\right]=g(z), \quad z \in D
$$

где $D$ - единичный квадрат, $g(z) \in A(D), \sigma_{m_{1}}, m_{2}(z)=z+m_{1} i+m_{2},\left|m_{1}\right|+\left|m_{2}\right|=2$, а неизвестная функция $\Omega(z) \in A(c D)$.

Библиография: 8 названий.

Введение. Пусть $R$-квадрат с вершинами $t_{1}=-t_{3}=(1+i) / 2, t_{2}=-t_{4}=(1-i) / 2$ и сторонами $l_{j}, j=\overline{1,4}$, пронумерованными в порядке положительного обхода границы $\partial R\left(t \in l_{1} \Rightarrow \operatorname{Im} t=-1 / 2\right)$. Множество $\left\{\sigma_{k}(z)\right\}: \sigma_{k}(z)=z+i^{k}, k=\overline{1,4}$, содержащее порождающие преобразования соответствующей двоякопериодической группы и преобразования, обратные к ним, обозначим через $I$. Эти преобразования переводят $R$ в четыре конгруэнтных квадрата $R_{k}$, имеющих с $R$ общую сторону. Квадраты $R_{k}$ образуют "первое окаймление" $R$. Преобразования группы, переводящие $R$ в конгруэнтные квадраты, которые имеют общие стороны уже не с $R$, а с квадратами из первого окаймления, обозначим через $I I$. Совокупность таких квадратов назовем "вторьм окаймлением" и т. д. Второе окаймление ограничивает конечную крестообразную область $D$, состоящую из исходного квадрата и его первого окаймления. Цель статьи - исследование линейного разностного уравнения с постоянными коэффициентами

$$
(V \Omega)(z) \equiv \sum_{\sigma_{k} \in I I} \Omega\left(\sigma_{k}(z)\right)=g(z), \quad z \in R .
$$

Предполагаем, что функция $g(z) \in A(D)[1]$ и $g(z) \in H_{\nu}(\partial R), 0<\nu \leqslant 1$ (т.е. удовлетворяет условию Гёльдера на $\partial R$ ). Решение ищется в классе четных функций, представимых в виде интеграла типа Коши

$$
\Omega(z)=\frac{1}{2 \pi i} \int_{\partial R} \frac{\varphi(\tau)}{\tau-z} d \tau, \quad z \notin \bar{R},
$$

плотность которого $\varphi(\tau) \in H_{\lambda}(\partial R)$. Класс таких четных плотностей обозначим через $B$. Другими словами, функция $g(z)$ голоморфна в $D$, а решение $\Omega(z)-$ вне $R$, причем $\Omega(\infty)=0$. Множество

$$
A=C \backslash \bigcup \sigma_{k}(R)
$$


не является связным. Уравнение (1) выполняется только на одной связной компоненте $D$, содержащей $R$, что не позволяет применить к нему классические методы исследования операторов свертки (см. [2] или [3]). При этом функция $g(z)$ не обязана быть аналитически продолжимой через какой-нибудь отрезок границы $\partial D$.

На стороне $l_{k}$ введем функцию $\alpha(t)=\sigma_{k}(t), k=\overline{1,4}$. Получим сдвиг $\alpha(t): \partial R \rightarrow \partial R$, изменяющий ориентацию, причем $\alpha(\alpha(t)) \equiv t, t \notin\left\{t_{k}\right\}$. Плотность интеграла $(2)$ определена с точностью до слагаемого $a^{+}(\tau)$ и за счет подбора голоморфной в $R$ функции $a(z)$ потребуем, чтобы

$$
\varphi(\tau)+\varphi(\alpha(\tau))=0
$$

- см. теорию краевой задачи Карлемана [4]. Заметим, что уравнение (1) по первому окаймлению, т.е. в случае, когда сумма берется по четырем преобразованиям из $I$, было исследовано ранее автором в статье [5]. Его приложения к проблеме моментов Стилтьеса указаны в [6].

В п. 1 доказано, что однородное уравнение

$$
(V \Omega)(z)=0, \quad z \in R
$$

имеет только тривиальное решение. Найдены также условия разрешимости неоднородной задачи.

В п. 2 рассмотрена проблема моментов для целых функций экспоненциального типа (ц.ф.э.т.)

$$
\int_{0}^{\infty} F(x) x^{4 k} e^{-x}\left(\cos x+e^{-x}\right) d x=c_{k}, \quad k=0,1, \ldots
$$

С помощью преобразования Бореля $[7$, гл. $1, \S 1$, п. 1] проблема моментов (6) сводится к исследованию разностного уравнения (1).

1. Введем еще один сдвиг, определив его на стороне $l_{k}$ формулой $\alpha_{1}(t)=t-i^{k}$, $k=\overline{1,4}$. Ясно, что $\alpha_{1}(t): \partial R \rightarrow \partial D$. Для интеграла

$$
\Phi(z)=\frac{1}{\pi i} \int_{\partial R} \varphi(\tau) E(\tau-z) d \tau
$$

с ядерной функцией

$$
E(z)=\sum_{\sigma \in I I}(\sigma(z))^{-1}
$$

справедлив аналог формулы Сохоцкого $\Phi^{+}\left(\alpha_{1}(t)\right)=-\varphi(\alpha(t))+\Phi(t)$, если только $z \rightarrow$ $\alpha_{1}(t), z \in D, t \in \partial R$. С помощью интегрального представления (2) разностное уравнение (1) запишется в виде функционального уравнения

$$
\Phi^{+}\left(\alpha_{1}(t)\right)=g^{+}\left(\alpha_{1}(t)\right), \quad t \in \partial R
$$

Заменим в соотношении (8) $t$ на $\alpha(t)$ и полученное равенство выгтем из исходного. Получим интегральное уравнение

$$
(T \varphi)(t) \equiv \varphi(t)+\frac{1}{2 \pi i} \int_{\partial R} K(t, \tau) \varphi(\tau) d \tau=g^{+}\left(\alpha_{1}(t)\right)-g^{+}\left(\alpha_{1}(\alpha(t))\right)
$$


с ядром

$$
K(t, \tau) \equiv E\left(\tau-\alpha_{1}(t)\right)-E\left(\alpha(\tau)-\alpha_{1}(\alpha(t))\right)
$$

Сразу отметим два обстоятельства. Первое состоит в том, что ядро (10) ограничено на $\partial R$, т.е. уравнение $(9)$ - это уравнение ядра непосредственно проверяется перебором различных вариантов взаимного расположения точек $\tau$ и $t$ на сторонах квадрата. Второе обстоятельство заключается в том, что если доопределить сдвиг $\alpha(t)$, формально определенньй пока только на $\partial R$, в квадратах из первого окаймления аналитическим продолжением с соответствующей стороны $\partial R$ и получить кусочно-линейную функцию $\alpha(z)$, то $\alpha_{1}(\alpha(t)) \neq \alpha\left(\alpha_{1}(t)\right)$. Кроме того, $\alpha\left(\alpha_{1}(t)\right) \equiv t, \alpha_{1}(i t)=i \alpha_{1}(t), \alpha(i t)=i \alpha(t)$.

Займемся исследованием однородного уравнения $Ф$ редгольма

$$
(T \varphi)(t)=0 \text {. }
$$

Для этого введем банахово пространство $\widetilde{C}(\partial R)$ - множество четных функций $\varphi(t) \in$ $C\left(\bar{l}_{k}\right), k=\overline{1,4}$, с нормой $\|\varphi\|=\max |\varphi(t)|, t \in \partial R$. Очевидно, что $T: \widetilde{C}(\partial R) \rightarrow \widetilde{C}(\partial R)$. В силу четности $\varphi(t)$ ядро (10) можно заменить новым ядром

$$
\widetilde{K}(t, \tau)=2^{-1}(K(t, \tau)-K(t,-\alpha(\tau))) .
$$

Оценим модуль ядра (12), причем в силу симметрии считаем без ограничения общности, что $t \in l_{1}$. Возможны три варианта.

a) Случай $\tau \in l_{1}$. Тогда, вводя обозначение $w=\tau-t$, имеем

$$
\tau-\alpha_{1}(t)=w+i, \quad \alpha(\tau)-\alpha\left(\alpha_{1}(t)\right)=w-i, \quad w \in[-1 ; 1] .
$$

Ядро (10) запишется в виде $K(t, \tau)=2 i f\left(w^{2}\right)$, где

$$
f(x)=2\left\{\frac{3-x}{(x+1)(x+9)}-\frac{x+5}{x^{2}-6 x+25}-\frac{2(x+5)}{x^{2}+6 x+(-25)}\right\} .
$$

Так как $f^{\prime}(x)<0$ при $x \in[0 ; 1]$, то

$$
|\widetilde{K}(t, \tau)| \leqslant f(0)-f(1) \leqslant \frac{37}{60} .
$$

б) Случай $\tau \in l_{3}$. В данном случае

$$
\tau-\alpha_{1}(t)=w+i, \quad \alpha(\tau)-\alpha\left(\alpha_{1}(t)\right)=w-3 i, \quad w=i+\vartheta, \quad \vartheta \in[-1 ; 1] .
$$

Поэтому при вычислении ядра (10) получим $K(t, \tau)=-2 i f\left(\vartheta^{2}\right)$. Далее,

$$
f(x)=\frac{4}{x+16}+\frac{4(x+8)}{x^{2}+64}+\frac{2(x+2)}{x^{2}+4}+\frac{6(x+10)}{x^{2}+16 x+100}, \quad x \in[0 ; 1] .
$$

Правая часть формулы (13) представляет собой сумму четырех слагаемых, которые обозначим соответственно через $b_{j}(x), j=\overline{1,4}$. При $x_{1} \in[0 ; 1]$ имеем $\left|b_{j}(x)-b_{j}\left(x_{1}\right)\right| \leqslant b_{j}$; $b_{1}=1 / 68, b_{2}=7 / 130, b_{3}=1 / 4, b_{4}=7 / 185$, т.е.

$$
|\widetilde{K}(t, \tau)| \leqslant 0.363 \text {. }
$$


в) Случай $\tau \in l_{4}$. Тогда

$$
\begin{aligned}
& \tau=i \nu-\frac{1}{2}, \quad \nu \in\left[-\frac{1}{2} ; \frac{1}{2}\right], \quad t=\mu-\frac{i}{2}, \quad \mu \in\left[-\frac{1}{2} ; \frac{1}{2}\right], \\
& \tau-\alpha_{1}(t)=\tau-t+i, \quad \alpha(\tau)-\alpha\left(\alpha_{1}(t)\right)=\tau-t+1-2 i .
\end{aligned}
$$

Введя обозначение $\gamma=i \nu-\mu$, запишем ядро (10) в виде

$$
\begin{aligned}
K(t, \tau)= & \frac{1-7 i}{\gamma^{2}+12+7 i / 2}+\frac{5-3 i}{\gamma^{2}-4+15 i / 2}-\frac{3(1+i)}{\gamma^{2}-9 i / 2} \\
& +\frac{3-i}{\gamma^{2}-2+3 i / 2}-\frac{1+5 i}{\gamma^{2}+6-5 i / 2}+\frac{3-5 i}{\gamma^{2}+4+15 i / 2} .
\end{aligned}
$$

Слагаемые в правой части (14) обозначим через $I_{j}(\gamma), j=\overline{1,6}$ соответственно. Если $\gamma_{1}=i \nu_{1}-\mu_{1}$ и $\nu_{1}, \mu_{1} \in[-1 / 2 ; 1 / 2]$, то $\left|I_{j}(\gamma)-I_{j}\left(\gamma_{1}\right)\right| \leqslant a_{j}$. Здесь $a_{1}=0.05, a_{2}=0.12$, $a_{3}=0.3, a_{4}=0.33, a_{5}=0.12, a_{6}=0.12$. Итак,

$$
|\widetilde{K}(t, \tau)|<0.56 \text {. }
$$

Такая же оценка вьполняется и при $\tau \in l_{2}$. Из полученных оценок модуля ядра следует, что уравнение $(11)$ не имеет нетривиальных решений $\varphi(t) \in \widetilde{C}(\partial R)$.

Пусть

$$
g^{+}\left(\alpha_{1}(t)\right) \in B \Longleftrightarrow g^{+}\left(\alpha_{1}(\alpha(t))\right) \in B .
$$

В силу свойств ядра (10) уравнение (9) будет иметь единственное решение $\varphi(t) \in B$. Кроме того, решением уравнения (9) является и функция $\psi(t)=-\varphi(\alpha(t))$ - достаточно в $(9)$ заменить $\tau$ и $t$ на $\alpha(\tau)$ и $\alpha(t)$ соответственно. Поэтому разность $\varphi(t)-\psi(t)$ удовлетворяет (11), т.е. выполнено (4).

Теперь подчеркнем принципиальную разницу между разностным уравнением по "первому окаймлению" и рассматриваемым разностньг уравнением (1) по "второму окаймлению". Для сведения первого из них к уравнению Фредгольма второго рода использовалась краевая задача Карлемана $\Phi^{+}(t)= \pm \Phi^{+}(\alpha(t))$, заданная на всем контуре $\partial R$. Поэтому обратньй переход от уравнения $\Phi$ редгольма к разностному уравнению мог привести лишь к уравнению $(V \Omega)(z)=g(z)+c, z \in R$. Сказанное справедливо в предположении, что уравнение $\Phi$ редгольма разрешимо. Доказательство этой разрешимости и представляло основную трудность в [5]. Но если данная трудность была преодолена, то выяснить, когда $c=0$, было уже не сложно.

Для рассматриваемого уравнения (1) дело обстоит совсем иначе. Хотя интегральное уравнение (9) безусловно разрешимо, но интеграл типа Коши (2) может удовлетворять разностному уравнению $(V \Omega)(z)=g(z)+\vartheta(z)$. Здесь $\vartheta(z) \in A(D)$, причем $\vartheta^{+}\left(\alpha_{1}(t)\right)=\vartheta^{+}\left(\alpha_{1}(\alpha(t))\right), t \in \partial R$. Множество таких функций “достаточно мощно", поскольку множество $\alpha_{1}(\partial R)$ является только частью границы $\partial D$. Запишем окончательный результат.

ТЕорема 1. Линейное разностное уравнение (1) разрешимо тогда и только тогда, когда выполнено счетное число условий разрешимости

$$
\frac{1}{2 \pi i} \int_{\partial R} \varphi(\tau) E^{(2 j)}(\tau) d \tau=g^{(2 j)}(0), \quad j=0,1, \ldots
$$

Здесь $\varphi(t)$ - единственное решение уравнения (9) из класса В. Однородное уравнение (5) имеет только тривиальное решение. 
2. Пусть $F(z)$ - ц.ф.э.т., ассоциированная по Борелю с решением $\Omega(z)$ разностного уравнения (1). Тогда

$$
\Omega(z)=\int_{0}^{\infty} F(x) \exp (-z x) d x, \quad \operatorname{Re} z>\frac{1}{2} .
$$

Из структуры ядра (10) и свойств преобразования Бореля следует, что если $g(i z)=g(z)$, то

$$
\Omega(i z)=\Omega(z) \Longleftrightarrow F(i z)=-i F(z) .
$$

Считаем, что для данной функции $g(z)$ уравнение (1) разрешимо, т.е. выполнены условия (15). Вместо (1) получим функциональное уравнение

$$
\int_{0}^{\infty} F(\tau)\left[4 e^{-\tau} \cos \tau \operatorname{ch} z \tau+2 e^{-2 \tau}(\cos z \tau+\operatorname{ch} z \tau)\right] d \tau=g(z), \quad z \in R .
$$

Сразу заметим, что

$$
\int_{0}^{\infty} F(x) x^{4 k+2} e^{-x} \cos x d x=0, \quad k=0,1, \ldots
$$

Действительно, индикатор и тип ц.ф.э.т. $F(x)$ вычисляются по формулам

$$
h_{F}(\vartheta)=\frac{\cos \vartheta+\sin \vartheta}{2}, \vartheta \in\left[0 ; \frac{\pi}{2}\right], \quad h_{F}\left(\vartheta+\frac{\pi}{2}\right)=h_{F}(\vartheta), \quad \sigma_{F}=\frac{\sqrt{2}}{2}
$$

Пусть $L_{R}$ - контур, образованный отрезком [0; $\left.R\right]$, дугой $z=R \exp (i \vartheta), \vartheta \in[0 ; \pi / 2]$, и отрезком мнимой оси $[-R i ; 0]$. Тогда

$$
\begin{aligned}
& \lim _{R \rightarrow \infty} \int_{L_{R}} F(z) z^{4 k+2} \exp ((i-1) z) d z \\
& \quad=\int_{0}^{\infty} F(x) x^{4 k+2} \exp ((i-1) x) d x-\int_{0}^{\infty} F(i y)(i y)^{4 k+2} \exp ((i-1) i y) i d y=0
\end{aligned}
$$

откуда и вытекает (17) - см. с незначительными изменениями схему, изложенную в [8, гл. $3, \S 3$, п. 1].

Тогда из (16) следует $(6)$, причем $c_{k}=g^{(4 k)}(0) / 4$.

Обратно, пусть рассматривается проблема моментов $(6)$ в классе $\widetilde{B}$ ц.ф.э.т. $F(z)$, имеющих нижние функции (2) с плотностью из $B$. Введем степенной ряд

$$
g(z)=\sum_{k=0}^{\infty} \frac{c_{k} z^{4 k}}{(4 k) !}
$$

Если $g(z) \in A(D)$ и $g^{+}\left(\alpha_{1}(t)\right) \in B$, то исследование проблемы моментов (17) сводится к исследованию разностного уравнения (1). Ограничения на $g(z)$ заведомо выполнены, если у степенного ряда радиус сходимости $r>\sqrt{5 / 2}$. Последнее неравенство легко проверить. В частности, класс $\widetilde{B}$ является классом единственности интерполяционной задачи (6). Сформулируем окончательньй результат.

ТЕОрема 2. Пусть функиия (18) удовлетворяет всем условиям, наложсенным ранее на коэффиииент $g(z)$ уравнения (1). Тогда неоднородная проблема моментов (6) разрешима тогда и только тогда, когда для единственного решения уравнения (9) со свойством (4) выполнены условия разрешимости (15). 


\section{СПИСОК ЦИТИРОВАННОЙ ЛИТЕРАТУРЫ}

[1] Хавин В.П. Пространства аналитических функций // Итоги науки и техники. Математический анализ 1964 г. М.: ВИНИТИ, 1966. С. 76-174.

[2] Напалков В. В. Уравнения свертки в многомерных пространствах. М.: Наука, 1982.

[3] Коробейник Ю. В. О решениях некоторых функционалных уравнений в классе функций, аналитических в выпуклых областях // Матем. сб. 1968. Т. 75. С. 225-234.

[4] Чибрикова Л.И. О граничных задачах для прямоугольника // Ученые зап. Казанского ун-та. 1964. Т. 123. № 9. С. 15-39.

[5] Гарифьянов $\Phi . Н$. Проблема обращения особого интеграла и разностные уравнения для функций, аналитических вне квадрата // Изв. вузов. Сер. матем. 1993. № 7. С. 7-16.

[6] Гарифьянов Ф. Н. Моменты Стильтьса целых функций экспоненциального типа // Матем. заметки. 2000. Т. 67. № 5. С. 674-679.

[7] Бибербах Л. Аналитическое продолжение. М.: Наука, 1967.

[8] Титчмарш Е. Теория функций. М.: Наука, 1980.

Казанская государственная

Поступило

сельскохозяйственная академия

18.05 .2000 